\section{Inquéritos domiciliares de saúde: potencialidades e desafios}

\section{Health household surveys: potentials and challenges}

\section{Resumo}

É apresentada uma síntese do desenvolvimento das medidas de estado de saúde de populações e do reconhecimento da necessidade de inquéritos populacionais periódicos para a geração dos novos indicadores. Traça-se um breve histórico sobre o desenvolvimento dos inquéritos, apontando conjunturas favoráveis no Brasil para o estabelecimento de uma matriz de diferentes modalidades de inquéritos que passem a integrar de forma consistente $o$ sistema de informação em saúde do país. Destacam-se algumas questões metodológicas relativas aos inquéritos e apontase o acúmulo de investimento já feito no país em validação e avaliação de instrumentos e escalas e em desenhos amostrais, entre outros aspectos. É enfatizado que a monitorização da equidade em saúde deve ser uma atribuição central dos inquéritos nacionais, considerando o patamar prevalente de concentração da renda, o que implica um conjunto de definições e escolhas de variáveis e indicadores. Ressalta-se que as linhas de pesquisa relativas aos inquéritos e à sua aplicação na análise das desigualdades em saúde podem constituir espaços significativos para o desenvolvimento de teorias epidemiológicas e de uma prática afinada com o campo da Saúde Coletiva.

Palavras-chave: Inquéritos domiciliares de saúde. Indicadores de estado de saúde. Sistema de informação de saúde. Desigualdades sociais em saúde.
Marilisa Berti de Azevedo Barros

Departamento de Medicina Preventiva e Social/FCM/UNICAMP

E-mail:marilisa@unicamp.br 


\section{Abstract}

This paper presents a short description of the development of measures for population health conditions, focusing on the recognition of periodic population surveys to provide new indicators. It also provides brief history of the development of population surveys, and points out some favorable conjunctures in Brazil for establishing a matrix of several types of surveys that could consistently integrate the Brazilian Health Information System. Several methodological issues related to the development of surveys are discussed, and the investments already made in the validation and evaluation of tools, scales, and sample design are stressed. Considering the existence of high concentration of wealth, health equality surveillance is emphasized as a central responsibility of nationwide surveys, which calls for the definition of variables and indicators. This paper highlights that research lines related to surveys and health inequality may establish significant spaces for the development of epidemiological theories and a more integral practice in the Collective Health arena.

Keywords: Household interview surveys. Health status indicators. Health information system. Social inequalities in health.

\section{As dimensões do estado de saúde}

O monitoramento do estado de saúde das populações é fundamental para a formulação e avaliação das políticas e programas de saúde ${ }^{1}$, sendo de interesse de todos os níveis de governo, e também da sociedade em geral e suas organizações, na busca de melhores patamares de saúde.

O estado de saúde tem sido entendido como fortemente influenciado pelo contexto econômico e social e mais diretamente determinado por quatro vertentes de fatores: a biologia humana, o meio ambiente, o estilo de vida e o sistema de atenção à saúde. Os serviços de saúde, dialeticamente, constituem também a resposta socialmente organizada para fazer face ao estado de saúde prevalente ${ }^{2}$. Evans e Stoddart ${ }^{3}$ diferenciam, entre os elementos constitutivos do estado de saúde, os componentes "doença" " "saúde e funcionamento", discriminando, desta forma, a visão clínica (necessária às condutas médicas) da percepção das próprias pessoas sobre os múltiplos aspectos que conformam a sua saúde. Estes autores entendem que a busca da saúde e da adequada organização dos cuidados deve visar a obtenção do bem-estar, componente que tem sido pouco operacionalizado por se tratar de dimensão complexa com valores substantivos que envolvem, segundo Griffin ${ }^{4}$, realização, profundidade das relações pessoais, satisfação, entendimento e dignidade humana.

O estado de saúde passa a ser concebido como constituído por subdimensões que respondem ao avanço e à complexidade do conceito de saúde-doença, e esses componentes seriam captados por diferenciados indicadores e distintos métodos de mensuração.

A avaliação do estado de saúde das populações dependeu, durante longo período de tempo, quase que exclusivamente dos indicadores derivados de dados de óbitos, mas estes índices perderam progressivamente a capacidade de medir a saúde em decorrência da forte redução das taxas de mortalidade, com os conse- 
qüentes aumentos da sobrevida e da prevalência de doenças crônicas, que mesmo de baixa letalidade podem ser altamente incapacitantes. O novo espectro de problemas de saúde passou a requerer, para a adequada mensuração, a construção de indicadores de incidência e prevalência de doenças e das lesões provocadas por acidentes e violências. A presença simultânea de várias doenças no mesmo indivíduo, fenômeno que se intensifica com o aumento da sobrevida da população, demandou a proposição de índices de comorbidades ${ }^{5}$.

O novo contexto também deixa claro que ter uma doença diagnosticada não se acompanha necessariamente do mesmo grau de prejuízo nos níveis de saúde e de desempenho das tarefas cotidianas. O "grau" de saúde dos pacientes diagnosticados dependerá da magnitude com que a doença afeta as suas atividades e o seu bem-estar. O impacto da doença em provocar limitações e incapacidades passa a ser dimensionado por instrumentos que avaliam a preservação das atividades da vida diária (básicas e instrumentais) e da qualidade de vida relacionada à saúde. Estes últimos implicam na quantificação de aspectos subjetivos da percepção e avaliação das pessoas sobre a própria condição de saúde-doença e diferenciam-se entre os que objetivam a análise global do estado de saúde e aqueles dirigidos a avaliar di- mensões específicas, tais como: bem-estar psíquico, bem-estar social, dor, incapacidades físicas e outras ${ }^{6}$.

Observa-se, neste processo, uma ampliação significativa do conceito de saúde e do espectro de indicadores que passam a ser necessários para o monitoramento da saúde (Quadro 1), sendo que os novos indicadores não substituem mas são complementares aos anteriormente utilizados.

Para dar conta da avaliação das várias dimensões do estado de saúde, inúmeros instrumentos foram desenvolvidos. Muitos dos questionários e escalas elaborados para a avaliação da qualidade de vida relacionada à saúde foram elaborados no âmbito da investigação clínica. Os indicadores passaram a incorporar, e com ampla aceitação, elementos subjetivos de auto-avaliação. Para tanto, houve a necessidade do desenvolvimento de bases teóricas sobre psicofísica e psicometria que pudessem respaldar o uso de julgamento subjetivo como forma adequada de mensuração da saúde ${ }^{6}$.

Um corpo de informações válidas passa a ser cada vez mais requisitado para as decisões, seja na clínica ou nas intervenções em saúde coletiva. E os indicadores de saúde, que se distanciavam entre os utilizados na abordagem do paciente e os utilizados no campo da Saúde Pública, passam a ter aplicações intercambiadas.

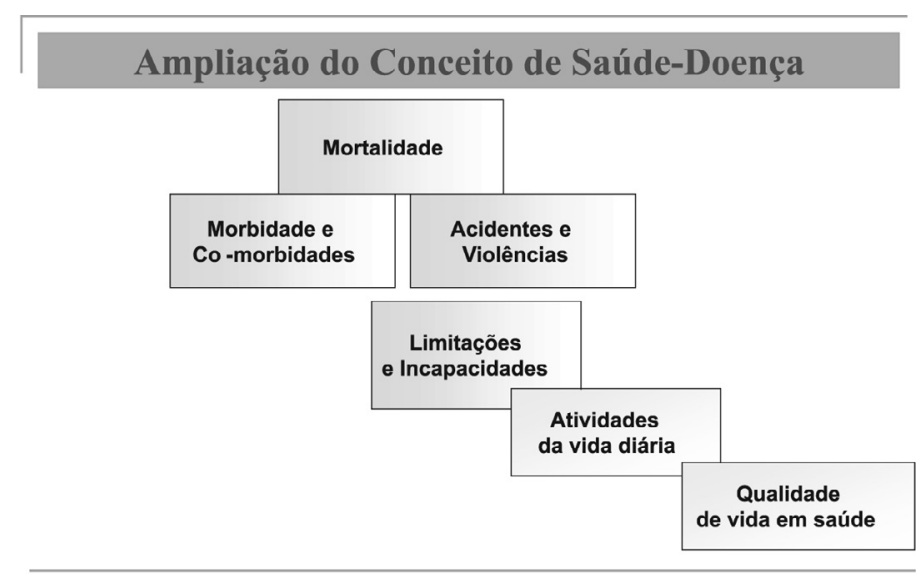

Quadro 1 - Ampliação do Conceito de Saúde-Doença

Picture 1 - Widening of the Health-Disease Concept 
As várias dimensões da saúde e da qualidade de vida em saúde passam a ser escrutinadas e quantificadas com instrumentos que são validados e traduzidos para diferentes idiomas. Uma profusão destes instrumentos abarca os mais diversificados aspectos da saúde, incluindo até os relativos à vida espiritual ${ }^{7}$ e ao funcionamento e satisfação sexual ${ }^{8}$ e valorizando temas como felicidade e bem-estar. A quantidade de instrumentos já desenvolvidos pode ser apreciada no Quadro 2, que apresenta o número de questionários e escalas sobre diferentes tópicos que foram, ainda na década de 90, selecionados e avaliados por McDowell e Newell ${ }^{6}$.

A quantidade de domínios que podem ser abarcados por um único instrumento é exemplificada pelas oito dimensões contidas no Medical Outcome Studies - 36 item Short Form Health Survey (SF-36): capacidade funcional, aspectos físicos, dor, estado geral de saúde, saúde mental, vitalidade, aspectos sociais e aspectos emocionais ${ }^{9}$.

Entretanto, os autores têm enfatizado a dificuldade de comparação de informações baseadas em percepções e relatos, pois elas sofrem forte influência dos contextos culturais, o que pode ser detectado por indícios de importante inconsistência em resultados obtidos de diferentes países ${ }^{10}$.
Além da avaliação do estado de saúde, o dimensionamento dos determinantes referentes às condições de vida e aos fatores de risco para doenças crônicas é essencial para a análise de situação de saúde, e assume maior relevância quando políticas de promoção de saúde passam a ser efetivamente adotadas.

Como destacado, com a complexidade crescente do perfil das doenças prevalentes e da organização dos serviços, passou a ser requerida a mensuração das inúmeras dimensões da saúde. Para monitorar a situação de saúde das populações, os países passaram a utilizar matrizes de indicadores que necessariamente abrangem vários componentes do estado de saúde, incluindo também os determinantes e índices da qualidade da organização e do desempenho dos serviços.

A exigência dos novos indicadores está reconhecida nos modelos de avaliação dos sistemas de saúde utilizados em diferentes países e no modelo proposto pelo projeto Pro-Adess (Quadro 3) para a avaliação da performance do sistema de saúde brasileiro $^{11}$.

As novas modalidades de indicadores não derivam de fontes secundárias de dados, de modo que os sistemas nacionais de informação precisam progressivamente incorporar os inquéritos de base popu-

\section{Medindo o estado de saúde - Número de instrumentos avaliados por McDowell \& Newell, 1996}

- Limitações e Incapacidades Físicas - Activities of Daily living: 7 Instrumental Activities of Daily living: 9

- Saúde social: 13

- Bem estar psicológico: 10

- Depressão: 8

- Estado Mental: 11

- Dor : 9

- Estado geral de saúde e Qualidade de vida em saúde: 21

Fonte: McDowell \& Newell,1996

Quadro 2 - Medindo o estado de saúde

Picture 2 - Measuring health status 


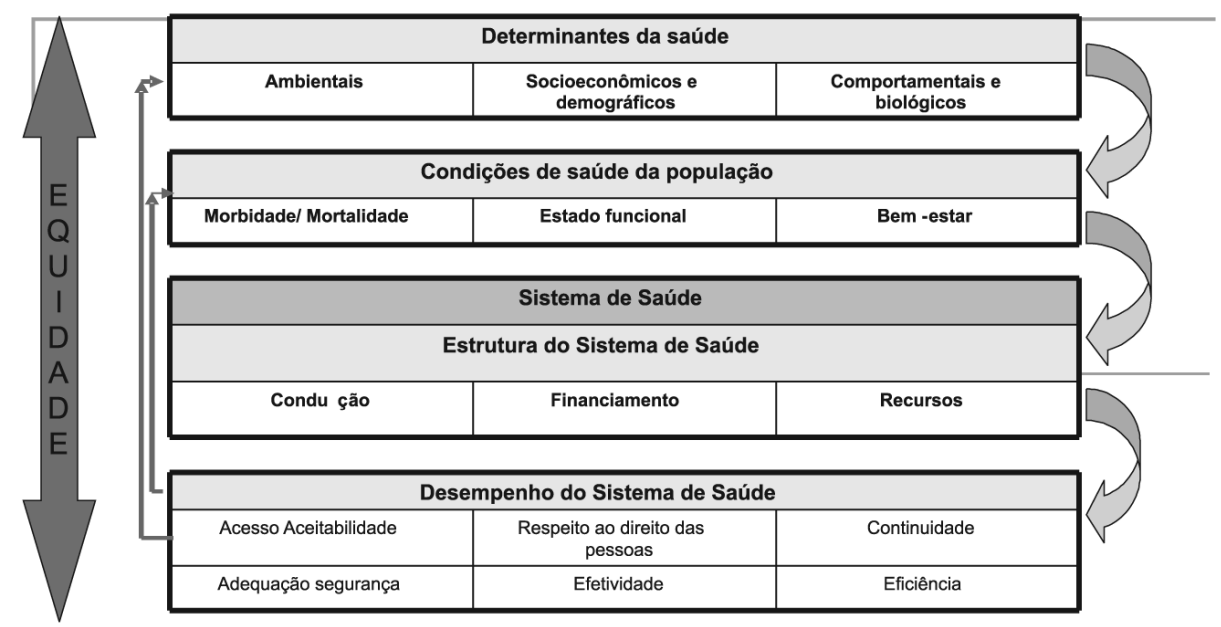

Fonte: Viacava et al, $2004^{11}$

Quadro 3 - Matriz de dimensões da avaliação de desempenho do Sistema de Saúde Picture 3 - Matrix of the dimensions of Health System performance assessment

lacional como um componente essencial para a geração de informações necessárias à formulação e avaliação das políticas sociais e intervenções do setor saúde ${ }^{1}$. A realização da Pesquisa Mundial da Saúde, promovida pela OMS em 71 países, incluindo o Brasil ${ }^{12}$, evidenciou a necessidade da realização de inquéritos populacionais para prover as informações requeridas para a avaliação de desempenho dos sistemas de saúde.

\section{Um breve histórico sobre os inquéritos de saúde}

Nos países desenvolvidos, os inquéritos de saúde de base populacional tiveram implementação mais significativa a partir dos anos 60 e, em períodos posteriores, passaram a ser aplicados com maior freqüência também nos países subdesenvolvidos. Em alguns destes países, com grande precariedade dos sistemas rotineiros de registros de dados, os inquéritos representam, muitas vezes, a única forma de obtenção de informação ${ }^{13}$.

Nos Estados Unidos, inquéritos perió- dicos de saúde foram iniciados no final da década de 50. O “National Health Interview Survey” (NHIS) foi realizado pela primeira vez em 1957 e, desde 1960, passou a ser aplicado anualmente pelo National Center for Health Statistics (NCHS), do Centers for Diasease Control and Prevention (CDC). Com o objetivo de monitorar as tendências de morbidades e de incapacidades, o conteúdo desse inquérito sofreu periódicas reformulações ${ }^{14}$.

Modalidade de inquérito que combina entrevistas com exames clínicos foi também implementada pelo NCHS, na mesma época em que teve início o NHIS. Os Health Examination Surveys (HES) foram realizados em 1960/62, 1963/65 e 1967/70 e, a partir de 1970, foram substituídos pelos National Health and Nutritional Examination Surveys (NHANES), que passam a ser contínuos em 1999. Os exames, que são realizados em unidades móveis, incluem avaliações médicas, odontológicas, medidas fisiológicas e testes laboratoriais. Vários estudos de seguimento vêm sendo acoplados a esses inquéritos ${ }^{15}$.

Pelo National Center for Chronic 
Disease Prevention and Health Promotion (NCCDPHP), também componente do CDC, passou a ser desenvolvido, desde 1981, o Behaviour Risk Factor Surveillance System (BRFSS), que constitui o maior sistema de vigilância por telefone realizado no mundo. Iniciado em 1981/83, passou a ser aplicado anualmente a partir de 1984, inicialmente em 15 Estados. Em 1994, todos os Estados americanos já estavam participando do sistema. O objetivo de criação do BRFSS foi obter dados de fatores de riscos para Estados e unidades geográficas menores, visto que o NCHS já obtinha essas informações, mas apenas com estimativas globais para o país ${ }^{16}$. Além destes, inúmeros outros inquéritos de grande porte, sobre os mais diferentes temas relativos à saúde, são periodicamente realizados nos Estados Unidos ${ }^{15}$.

No Reino Unido, que teve um inquérito de morbidade realizado em 1943/52 (Survey of Sickness), foi iniciada, em 1971, a aplicação anual do General Household Survey (GHS). Com o objetivo de permitir interrelacionar diferentes áreas de política social e monitorar mudanças, os resultados desse inquérito são muito utilizados pelo governo central e por profissionais e pesquisadores. A partir de 2000, o GHS passa a ser constituído por dois componentes: um contínuo e os trailers. O Reino Unido iniciou, em 1990, o National Statistics Omnibus Survey, com o objetivo de obter resultados rápidos sobre temas diversos como contraceptivos, medicamentos, cigarro, acesso à Internet, transporte, uso do tempo, mudança na renda familiar etc. Várias outras pesquisas são realizadas no Reino Unido, tais como inquéritos sobre estilo de vida, problemas específicos de saúde, saúde mental e percepção do público sobre o Sistema Nacional de Saúde (NHS). Em 1991, começou a ser aplicado o Health Survey for England, cobrindo a população adulta com 16 anos ou mais e, a partir de 1995, passando a também incorporar crianças na amostra. A cada ano, a pesquisa focaliza diferentes grupos demográficos, ou diferentes condições de saúde e de fatores de risco. A pesquisa combina questionários com medidas físicas. Inclui questões sobre saúde e indicadores psicossociais, demográficos e socioeconômicos; consumo de cigarro e álcool; indicadores de uso de serviços de saúde e de medicamentos e medidas de altura, peso e pressão arterial. $\mathrm{O}$ inquérito tem incluído mensurações de colesterol, fibrinogênio, hemoglobina e cotinina, entre várias outras ${ }^{17}$.

A Organização Mundial da Saúde (OMS) tem promovido, nas últimas décadas, várias pesquisas multicêntricas, envolvendo muitos países, sobre os mais diferentes temas de saúde. Algumas destas pesquisas incluem inquéritos de base populacional como aquela realizada sobre a prevalência de fatores de risco de doenças não transmissíveis ${ }^{18}$, e o estudo multicêntrico sobre comportamento suicida (ideação, plano e tentativa), que inclui inquérito de base populacional desenvolvido pelo Projeto SUPRE-MISS ${ }^{19}$.

Mais recentemente, a OMS organizou a realização do World Health Survey (WHS), aplicado em 71 países, entre os quais o Brasil, que tem por objetivo coletar informações para avaliar a performance dos sistemas de saúde. Esta iniciativa busca superar parte das críticas apresentadas ao indicador proposto pela OMS em $2000^{20}$.

Os inquéritos de base populacional têm sido freqüentemente realizados no Canadá e suas províncias, e na maioria dos países europeus, bem como em países em desenvolvimento, nestes, em geral, por iniciativa e propostas de agências e organizações internacionais ${ }^{21}$.

No Brasil, foi realizado na década de 70, em Ribeirão Preto, SP, um inquérito pioneiro de base populacional sobre morbidade referida e uso de serviços de saú$\mathrm{de}^{22}$. Esta iniciativa propiciou discussões sobre as diversas questões metodológicas afeitas a este tipo de pesquisa e estimulou o desenvolvimento de alguns projetos subseqüentes ${ }^{13,23,24}$. Em âmbito nacional, pesquisas com temas mais relacionados ao setor saúde iniciam-se em 1974/75, com o 
Estudo Nacional de Despesa Familiar (ENDEF) que foi seguido, na temática, pelas Pesquisas de Orçamento Familiar (POF) realizadas em 1987 e 1996. Voltadas para as questões de nutrição e reprodução, foram desenvolvidas a Pesquisa Nacional sobre Saúde e Nutrição (PNSN), em 1989, pelo Instituto Nacional de Alimentação e Nutrição (INAN) e Instituto Brasileiro de Geografia e Estatística (IBGE), e a Pesquisa Nacional sobre Demografia e Saúde (PNDS), implementada em 1996, sob os auspícios da Sociedade Bem-Estar Familiar no Brasil (BENFAM). Em 1996/97 foi realizada pelo IBGE a Pesquisa sobre Padrão de Vida (PPV), que cobriu 5.000 domicílios das regiões nordeste e sudeste, e incluiu várias questões de interesse da saúde. Pela cobertura territorial e periodicidade, revestem-se da maior importância no país as Pesquisas Nacionais por Amostras de Domicílios (PNADs), que tiveram início em $1967^{1}$. A potencialidade da PNAD para a realização de inquéritos de saúde foi identificada por Carvalheiro, na década de $70^{21}$. Em 1981, foi aplicado um primeiro suplemento de saúde na PNAD, seguido pelos realizados em 1986, 1988 e 1998 e $2003^{21,25}$.

Embora algumas questões sobre práticas preventivas e prevalência de comportamentos relacionados à saúde tenham sido incluídas em outros inquéritos, a primeira grande pesquisa nacional sobre esta temática é realizada pelo Instituto Nacional de Câncer, em 2002-2003, por demanda da Secretaria de Vigilância em Saúde do Ministério da Saúde ${ }^{26}$. Inovação significativa foi obtida com o desenvolvimento do sistema de vigilância de fatores de risco para doenças crônicas (VIGITEL), que inaugura no país o uso de inquéritos por telefone em âmbito nacional ${ }^{27}$.

Caminha-se, desta forma, no Brasil, para a consolidação de uma política que reconhece a necessidade de inquéritos periódicos para gerar informações que não são obtidas em registros contínuos e são essenciais para o planejamento e a avaliação das políticas de promoção de saúde e de prevenção e controle de agravos.

Experiências de âmbito menor que os inquéritos nacionais significam espaços e oportunidades fundamentais de experimentação e validação de instrumentos, de compreensão dos determinantes da variação dos valores médios nacionais ou regionais, e de aprofundamento de questões que necessitam de investigações mais específicas e detalhadas. Podem também atender a necessidades de gestão em nível municipal ou regional, não contempladas em pesquisas nacionais.

\section{Modalidades de inquéritos e algumas questões relativas a métodos e trabalho de campo}

Os inquéritos de base populacional, que usualmente constituem estudos de corte-transversal aos quais podem ser acoplados outros desenhos de pesquisas, podem ser classificados ou categorizados segundo um conjunto de características: abrangência geográfica, população/segmentos demográficos alvo, conteúdos temáticos, tipo de dado/material coletado, forma de obtenção da informação e periodicidade.

Quanto à temática dos inquéritos, que pode ser ampla e diversificada ou voltada para um tópico específico, vêm ganhando importância os conteúdos sobre os comportamentos relacionados à saúde ou os fatores de risco para doenças crônicas não transmissíveis. Entre estes fatores, pela reconhecida importância do efeito que exercem na morbimortalidade, são especialmente investigados o tabagismo, a dependência de álcool, a qualidade da dieta e o sedentarismo. A prevalência de condições crônicas é outro tópico relevante nos inquéritos e, entre as doenças que têm sido pesquisadas, destacam-se a hipertensão, o diabetes, os transtornos mentais e a presença de sobrepeso/obesidade, pelo maior impacto na demanda de serviços de saúde e na qualidade de vida dos pacientes.

Entre as questões metodológicas de grande interesse nos inquéritos encon- 
tram-se as que tratam dos modelos de amostragem, da qualidade e validade da informação e das taxas de não-resposta. Verifica-se que vários dos instrumentos desenvolvidos em outros países vêm sendo traduzidos e validados para uso na população brasileira. Uma breve listagem evidencia a diversidade de instrumentos trabalhados por diferentes instituições acadêmicas: o Alcohol Use Disorders Identification Test - AUDIT, ${ }^{28,29}$ Medical Outcome Studies - 36 item Short Form Health Survey SF36, ${ }^{30}$ Questionário Internacional de Atividade Física - QIAF, ${ }^{31}$ o Índice de Qualidade da Dieta - IQD, ${ }^{32}$ o Self Reporting Questionnaire -SR20 ${ }^{33}$ e a Escala de Apoio Social. ${ }^{34}$ Alguns pesquisadores brasileiros têm, também, validado informações obtidas dos entrevistados sobre algumas questões como: peso e altura ${ }^{35-39}$, presença de hipertensão $\operatorname{arterial~}^{40} \mathrm{e}$ informação materna sobre o peso dos filhos ao nascer ${ }^{41}$.

Outro aspecto relevante para a validade das estimativas geradas por um inquérito é o percentual de perdas. Mas, antes disto, é preciso considerar que inquéritos domiciliares não incluem os segmentos da população que estão institucionalizados ou que não têm domicílios, subgrupos que necessitariam de pesquisas especialmente desenhadas. Quanto ao percentual de nãoresposta, verifica-se que as altas taxas de violência urbana, verificadas hoje no Brasil, têm introduzido dificuldade adicional para a realização de inquéritos domiciliares.

Diversas estratégias têm sido utilizadas para melhorar o percentual de resposta: seleção e treinamento cuidadosos de entrevistadores; inserção de áreas residenciais de diferentes níveis econômicos já no estudo piloto; divulgação adequada para a comunidade por meio de cartas, mídia, unidades de saúde etc; apoio institucional com transporte mais seguro para áreas de maior risco; parceria com unidades básicas de saúde próximas aos setores sorteados, que têm vivência sobre acesso e receptividade e podem representar importante retaguarda em necessidades assistenciais; e flexibilidade do horário de trabalho dos entrevistadores para se adequar ao agendamento das entrevistas.

Os avanços nas questões metodológicas, gerando informações com melhor validade e estimativas com maior precisão, têm levado a ampliar a utilização dos resultados dos inquéritos de base populacional. Embora os inquéritos nacionais visem especialmente monitorar as condições de saúde das populações, é reconhecido que eles têm produzido um significativo avanço do conhecimento em amplo espectro de temas de saúde, gerando grande produção de artigos científicos.

Em síntese, os inquéritos de saúde possibilitam a obtenção de informações sobre diferentes dimensões do estado de saúde, sobre determinantes demográficos, sociais, econômicos e culturais da saúde-doença, além de comportamentos relacionados à saúde, muitos dos quais constituem conhecidos fatores de risco de doenças crônicas. Além disso, os inquéritos permitem conhecer a realidade do acesso aos múltiplos serviços e atividades da saúde. Em todas estas dimensões, os inquéritos possibilitam o reconhecimento e a análise do padrão das desigualdades sociais na saúde.

\section{Inquéritos e equidade em saúde}

As desigualdades sociais em saúde, registradas de longa data, tiveram seu estudo retomado com ênfase, na década de 90, frente à verificação da persistência ou ampliação dos diferenciais de saúde entre os segmentos sociais $^{42,43}$. A Organização Mundial da Saúde justificava sua força-tarefa no tema por considerar que as diferenças de saúde entre os estratos sociais eram excessivas e vulneráveis às tecnologias disponíveis. Além disso, reconheciam que os sistemas usuais de informação não permitiam monitorar as desigualdades e que o tema precisaria ser incluído nas agendas de gestores do sistema de saúde ${ }^{42}$.

Diferentes paradigmas e correntes de pensamento embasam as iniciativas científicas e as das agências internacionais que convergem suas atividades para o campo 
da eqüidade em saúde. Gwatkin ${ }^{43}$ identifica três correntes de pensamento/movimento no interior deste campo, que poderiam ser designados pelo seu enfoque central em pobreza e saúde, desigualdades em saúde e ineqüidades em saúde.

As análises das desigualdades sociais em saúde consubstanciam parte significativa do desenvolvimento da Epidemiologia Social nas três correntes identificadas por Krieger ${ }^{44}$ : Teoria Psicosocial, Produção Social da Doença e Epidemiologia Eco-social/Multinível. As três vertentes diferenciam-se na ênfase com que as dimensões política e econômica são consideradas $^{44,45}$. Os estudos sobre equidade alinham-se às demandas do campo da Saúde Pública/Saúde Coletiva e respondem, de certa forma, às críticas feitas à trajetória da epidemiologia na segunda metade do século $\mathrm{XX}^{46,47}$.

Nas últimas décadas, os estudos de desigualdades sociais em saúde ganharam aporte significativo com o desenvolvimento de novos métodos e desenhos de estudos, com a diversificação dos eventos de saúde que passam a constituir objeto de análise, com a utilização de técnicas alternativas de mensuração das disparidades e com o acúmulo de conhecimentos sobre os principais vieses a que os estudos estão sujeitos $^{48,49}$. Para quantificar e monitorar a disparidade social em saúde, inúmeras medidas são propostas e ensaiadas, incluindo medidas absolutas ou relativas, de efeito ou de impacto, brutas ou modeladas $^{50,51}$

Quanto à configuração dos estratos socioeconômicos ou de classes sociais, a ocupação ganha espaço destacado, sendo considerada "a core socioeconomic variable"52. Entre as classificações de ocupações, são diferenciadas as hierárquicas das estruturais, estas últimas com orientação marxista ou filiação mais eclética; ${ }^{50}$ alguns pesquisadores têm apontado a importância da análise de classes sociais nos estu-

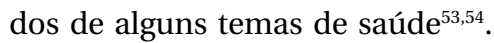

Além das iniqüidades em saúde relacionadas aos estratos socioeconômicos ou às frações de classe social, passam a ga- nhar foco e relevância as desigualdades referentes às diferenças de gênero, raça e etnia, área de moradia e afiliação religiosa, entre outros.

O monitoramento das desigualdades sociais em saúde, que em décadas anteriores restringia-se às taxas de mortalidade, amplia o espectro temático para incluir o perfil de morbidade e, em particular, a prevalência de fatores de risco de doenças crônicas. A literatura acumula evidências da maior prevalência de fumantes nos segmentos de baixa renda e escolaridade ${ }^{26} \mathrm{e}$, em especial, nos grupos mais excluídos e marginalizados ${ }^{55}$. São os estratos de pior nível socioeconômico que também concentram maiores prevalências de sedentarismo em contexto de lazer ${ }^{56,57}$, de uso abusivo ou dependência de álcool ${ }^{58,59}$, de maior ocorrência de obesidade ${ }^{60}$ e de dietas de pior qualidade ${ }^{32}$. Os segmentos economicamente desfavorecidos da população são os que apresentam maior vulnerabilidade à maioria dos comportamentos não saudáveis ${ }^{26,27,61}$. É reconhecido que uma fração substancial das diferenças sociais em morbidade e mortalidade decorrem das diferenças no padrão de comportamentos relacionados à saúde ${ }^{55,62}$. Os estudos apontam que os segmentos de melhor nível socioeconômico vêm adotando mais rapidamente estilos de vida mais saudáveis pelas condições materiais de vida que usufruem e pelo maior acesso à informação, situação que tende a resultar em ampliação das desigualdades sociais na morbimortalidade. Considerando o impacto dos comportamentos não saudáveis no perfil atual e futuro de saúde e a tendência de concentração destes comportamentos nos segmentos socialmente mais desfavorecidos, torna-se relevante monitorar as prevalências e identificar os subgrupos com maior vulnerabilidade, de forma a nortear políticas e programas de promoção da saúde e de controle de fatores de risco.

Estudos de morbidade referida também apontam as maiores prevalências nos segmentos de menor escolaridade ou ren- 
$\mathrm{da}^{24,26,63,64}$. A desigualdade social de acesso ao uso de serviços de saúde e às práticas preventivas é diferenciada conforme o tipo de serviço demandado. Para alguns serviços, a estruturação do SUS permitiu amplo acesso com forte impacto na redução das ineqüidades; para outros, persiste ainda nítido gradiente favorecendo os estratos sociais de maior escolaridade e ren$\mathrm{da}^{24,65,66}$. Para algumas práticas, entretanto, como a vacinação de idosos, verificase maior utilização pelos segmentos de menor nível socioeconômico que são, em maior proporção, cobertos pelo Sistema Único de Saúde ${ }^{67}$.

Para a análise da desigualdade social relativa a todos estes temas e ao amplo espectro das dimensões do estado de saúde, os inquéritos constituem estratégia apropriada para a análise e monitoramento da eqüidade em saúde ${ }^{68}$. Além da variedade de tópicos, os inquéritos possibilitam a análise das desigualdades entre segmentos sociais definidos segundo gênero, raça/ cor, renda, escolaridade, ocupação, indicadores compostos e área de moradia, entre outros.

Desafios para o aprimoramento dos inquéritos e sua integração no sistema nacional de informação de saúde

Reconhece-se que os inquéritos de base populacional são meios apropriados para a obtenção de muitos dos novos indicadores necessários para o monitoramento do estado de saúde e do desempenho dos sistemas de saúde, tendo como eixo a questão da eqüidade. O desenvolvimento de inquéritos acrescenta, entretanto, desafios à organização dos sistemas nacionais de informação de saúde ${ }^{1,25,69}$.

As informações geradas pelos inquéritos deveriam ser complementares, e sem sobreposições desnecessárias às derivadas de outros componentes do sistema de informação.

Por outro lado, várias modalidades de inquéritos nacionais revelam-se necessárias, o que exige a construção de uma ma- triz adequada e eficiente que viabilize monitorar as condições de saúde e o desempenho do sistema. A elaboração desta matriz implica a definição de conteúdos temáticos, indicadores básicos a serem gerados, abrangência geográfico-administrativa, populações alvo, periodicidades e métodos de coleta de dados, entre outros aspectos. A possibilidade de sobreposição de levantamentos e de obtenção de dados redundantes implica na exigência de verificação da real necessidade e complementaridade de cada tipo de pesquisa, especialmente em país com maior escassez de recursos.

A descentralização da gestão da saúde, muito significativa no sistema brasileiro coloca, em relação aos inquéritos, as questões do grau de validade da generalização de resultados e a pertinência da realização de inquéritos estaduais ou municipais, para atender necessidades dos diferentes níveis de gestão.

Os dados gerados pelos inquéritos, assim como pelos demais componentes do sistema de informação de saúde, precisarão ser oportunos e atuais, além de acurados e completos.

A qualidade das informações geradas pelos inquéritos é um desafio central que aponta para a necessidade de implementar ou propiciar maior desenvolvimento de várias iniciativas. Uma das atividades relevantes para o aprimoramento dos inquéritos é o desenvolvimento, a validação e a avaliação de questionários, escalas e instrumentos, visando comparações com dados internacionais, mas com uma perspectiva crítica de pertinência frente às necessidades e prioridades nacionais.

Quanto às questões metodológicas, inúmeros aspectos requerem desenvolvimentos e aprimoramentos: processos de coleta das informações, meios de obtenção dos dados, uso de computador em campo, treinamentos de entrevistadores, estratégias de redução da não-resposta, entre outros.

Algumas questões éticas precisam ser consideradas em relação aos inquéritos. É 
necessária uma discussão sobre formas de disponibilizar bancos de dados de inquéritos, realizados por instituições públicas ou financiados com recursos públicos, a pesquisadores e gestores interessados, certamente preservando a identidade dos entrevistados. Inclusive, como é usual para algumas agências ${ }^{15}$, a disponibilização de manuais e capacitações para o acesso efetivo aos dados.

Precisa ser feita uma discussão quanto à necessidade de carta de consentimento em inquéritos domiciliares que se baseiam exclusivamente em entrevistas, sem coleta de material biológico. Hoje, esta exigência se coloca inclusive para um estudo de natureza qualitativa que se proponha, por exemplo, a entrevistar alguns secretários municipais de saúde.

Outros tópicos a considerar são o risco que envolve o trabalho dos entrevistadores, especialmente em situação de intensa violência urbana, e a atuação de entrevistadores e pesquisadores, frente a situações de necessidades médicas ou a eventos inusitados que podem ocorrer durante o trabalho de campo. As formas de retorno dos resultados para as pessoas entrevistadas também é questão que precisa ser considerada.
O grau extremo de concentração de renda do país e suas múltiplas conseqüências tornam o monitoramento das disparidades sociais uma atribuição essencial dos inquéritos de base populacional. Para responder a esta atribuição alguns aspectos são relevantes: seleção de indicadores de saúde sensíveis à influência das variáveis sociais, periodicidade apropriada para identificar mudanças significativas, formas de coleta da informação, escolha de medidas de desigualdades, e, em especial, de grupos de comparação (seleção de variáveis sociais e econômicas).

No âmbito disciplinar da epidemiologia, a realização de inquéritos de base populacional, pode propiciar o desenvolvimento de métodos, técnicas e conhecimentos que respondam com mais sintonia às necessidades do campo da Saúde Coletiva. As linhas de pesquisa voltadas ao desenvolvimento de inquéritos podem constituir espaços de formação de alunos de graduação e pós-graduação numa diretriz de compromisso com a saúde da população, e estimular a elaboração de teorias sobre a distribuição populacional das doenças, pouco desenvolvidas na Epidemio$\operatorname{logia}^{70}$, cuja vocação tem se voltado mais para a produção de evidências empíricas.

\section{Referências}

1. Viacava F. Informações em saúde: a importância dos inquéritos populacionais. Ciênc Saúde Coletiva 2002; 7(4): 607-21.

2. Castellanos PL. Epidemiologia, saúde pública, situação de saúde e condições de vida: considerações conceituais. In: Barata RCB, organizador. Condições de vida e saúde. Rio de Janeiro: Abrasco; 1997. pp. 31-75.

3. Evans RG, Stoddart GL. Producing health, consuming health care. Soc Sci Med 1990; 31(12): 1347-63.

4. Griffin J. A note on measuring well-being. In: Murray CJL et al. Summary measures of population health: concepts, ethics, measurement and applications. Geneva: WHO; 2002. pp. 129-33.

5. Charlson ME, Szatrowski TP, Peterson J, Gold J. Validation of a combined comorbidity index. J Clin Epidemiol 1994; 47(11): 1245-51.
6. McDowell I, Newell C. Measuring health: a guide to rating scales and questionnaires. New York: Oxford University Press; 1996.

7. Volcan SM, Sousa PL, Mari JJ, Horta BL. Relação ente bem-estar espiritual e transtornos psiquiátricos menores: estudo transversal. Rev Saúde Pública 2003; 37(4): 440-5.

8. Meston CM. Validation of the Female Sexual Functioning Index (FSFI) in women with female orgasmic disorder and in women with hypoactive sexual desire. J Sex Marital Ther 2003; 29(1): 39-46.

9. Ware JE, Kosinski M, Keller SD. SF-36 Physical and mental health summary scales: a user's manual. Boston: The Health Institute; 1994.

10. Sadana R, Mathers CD, Lopez AD, Murray CIL, Iburg KM. Comparative analyses of more than 50 household surveys on health status. In: Murray CJL et al. Summary measures of population health: concepts, ethics, measurement and applications. Geneva: WHO; 2002. pp. 369-86. 
11. Viacava F, Almeida C, Caetano C. et al. Uma metodologia de avaliação do desempenho do sistema de saúde brasileiro. Ciênc Saúde Coletiva 2004; 9(3): 711-24.

12. Szwarcwald CL, Viacava F. Pesquisa Mundial de Saúde no Brasil, 2003 (editorial). Cad Saúde Pública 2005; 21 Supl.: S4-S5.

13. Cesar CLG, Tanaka OY. Inquérito domiciliar como instrumento de avaliação de serviços de saúde: um estudo de caso na região sudoeste da área metropolitana de São Paulo, 1989-1990. Cad Saúde Pública 1996; 12 Supl. 2: 59-70.

14. Adams PF, Dey NA, Vickerie JL. Summary health statistics for the US population: National Health Interview Survey, 2005. Vital Health Stat 2007; 10 (233).

15. Zeni MB, Kogan MD. Existing population-based health databases: useful resources for nursing research. Nurs Outlook 2007; 55(1): 20-30.

16. $[\mathrm{CDC}]$ Centers for Disease Control and Prevention. National Center for Chronic Disease Prevention and Health Promotion. Behavioral risk factor surveillance system. Atlanta; 1999.

17. United Kingdom. Department of Health. Health Survey for England [on line]. Disponível em: URL: http:// www.dh.gov.uk/PublicationsAndStatistics/ PublishedSurvey?ListOfSurveySince1990/ GeneralSurveys/fs/en [Acessado em 27 de outubro de 2004].

18. Berrios X, Koponen T, Higuang T, Khaltaev N, Puska P, Nissinen A. Distribution and prevalence of major risk factors of non communicable diseases in selected countries: the WHO Inter - Health Programme. Bull World Health Organ 1997; 75(2): 99-108.

19. [WHO] World Health Organization. Multisite Intervention Study on Suicidal Behaviour SUPRE-MISS: Protocol of SUPRE-MISS. Geneva: WHO; 2002.

20. Almeida C, Braveman P, Gold MR, Szwarcwald CL, Ribeiro JM, Miglionico A et al. Methodological concern and recommendations on policy consequences of the World Health Report 2000. Lancet 2001; 357: 1692-7.

21. Barros, MBA. Introdução. In: César CLG, Carandina L, Alves MCGP, Barros MBA, Goldbaum M. Saúde e condição de vida em São Paulo: inquérito multicêntrico de saúde no Estado de São Paulo. São Paulo: USP/FSP, 2005.

22. Carvalheiro JR. Levantamento de condições de saúde por entrevistas domiciliárias [tese de livre-docência]. Ribeirão Preto: Faculdade de Medicina de Ribeirão Preto da USP; 1975.

23. Carandina L, Sanches O, Carvalheiro JR. Análise das condições de saúde e de vida da população urbana de Botucatu, SP. Descrição do plano amostral e avaliação da amostra. Rev Saúde Pública 1986; 20(6): 465-74.
24. Cesar CLG, Carandina L, Alves MCGP, Barros MBA, Goldbaum M, organizadores. Saúde e condição de vida em São Paulo. Inquérito multicêntrico de saúde no Estado de São Paulo- ISA-SP. (1ª ed.) São Paulo: FSP/ USP; 2005.

25. Viacava F, Dachs N, Travassos C. Os inquéritos domiciliares e o sistema Nacional de Informações em Saúde. Ciênc Saúde Coletiva 2006; 11(4): 863-9.

26. Brasil. Ministério da Saúde, SVS/SAS/INCA. Inquérito domiciliar sobre comportamentos de risco e morbidade referida de doenças e agravos não transmissíveis - Brasil: 15 capitais e Distrito Federal, 2002/2003. Rio de Janeiro: INCA; 2004.

27. Brasil. Ministério da Saúde, SVS/SGEP. Vigitel, Brasil, 2006. Vigilância de fatores de risco e proteção para doenças crônicas por inquérito telefônico. Brasília, DF; 2007.

28. Méndez BE. Uma versão brasileira do AUDIT - Alcohol use Disorder Identification Test. [dissertação de mestrado]. Pelotas: Universidade Federal de Pelotas; 1999.

29. Lima CT, Freire ACC, Silva APB, Teixeira RM, Farrel M, Prince M. Concurrent and construct validity of the AUDIT in an urban Brazilian sample. Alcohol Alcohol 2005; 40(6): 584-9.

30. Ciconelli, RM. Tradução para o Português e validação do questionário genérico de avaliação de qualidade de vida "Medical Outcome Studies, 36 - Item Short Form Health Survey (SF-36)" [tese de doutorado]. São Paulo: UNIFESP; 1997.

31. Matsudo S, Araújo T, Matsudo V, Andrade D, Andrade E, Oliveira LC , Braggion G. Questionário Internacional de Atividade Física (IPAQ): estudo de validade e reprodutibilidade no Brasil. Rev Bras Atividade Física e Saúde 2001; 6(2): 5-18.

32. Fisberg RM; Morimoto JM, Slater, B, Barros MBA, Carandina, L, BARROS, Goldbaum M, Latorre MRO, Cesar CLG. Dietary quality and associated factors among adults living in the state of São Paulo, Brazil. JADA 2006; 106 (12): 2067-72.

33. Mari JJ, Williams P. A validity study of a psychiatric screening questionnaire (SRQ-20) in primary care in the city of São Paulo. Br J Psychiatry 1986; 148: 23-6.

34. Griep RH, Chor D, Faerstein E, Werneck GL, Lopes CS. Validade de constructo de escala de apoio social de Medical Oucomes Study adaptada para o português no Estudo Pró-Saúde. Cad Saúde Pública 2005; 21(3): 703-14.

35. Schmidt MI, Duncan BB, Tavares M, Polancyk CA, Pellanda L, Zimmer PM. Validity of self-reported weighta study of urban Brazilian adults. Rev Saúde Pública 1993; 27(4): 271-6.

36. Chor D, Coutinho ESF, Laurenti R. Reliability of selfreported weight and height among state bank employees. Rev Saúde Pública 1999; 33: 16-23. 
37. Fonseca MJM, Faerstein E, Chor D, Lopes CS. Validity of self-reported weight and height and the body mass index within the "pró-saúde" study. Rev Saúde Pública 2004; 38(3): 1-7.

38. Oliveira AF, Gadelha AMJ, Leal MC, Szwarcwald CL. Estudo da validação de peso e estatura em gestantes atendidas em maternidade municipal no Rio de Janeiro, Brasil. Cad Saúde Pública 2004; 20(Supl.1): S92-S100.

39. Silveira EA, Araújo CL, Gigante DP, Barros AJD, Lima MS. Validação do peso e altura referidos para o diagnóstico do estado nutricional em uma população de adultos no Sul do Brasil. Cad Saúde Pública 2005; 21(1): 235-45.

40. Lima-Costa MF, Peixoto SV, Firmo JDA. Validade da hipertensão arterial auto-referida e seus determinantes (projeto Bambui). Rev Saúde Pública 2004; 38(5): 637-42.

41. Victora CG, Barros FC, Martines JC, Beria JU, Vaughan JP. As mães lembram o peso ao nascer de seus filhos? Rev Saúde Pública 1985; 19(3): 195-200.

42. [WHO] World Health Organization. Equity in health and health care: a WHO/SIDA initiative. Geneva: WHO; 1996.

43. Gwatkin DR. Health inequalities and the health of the poor: What can we do? Bull World Health Organ 2000; 78(1): 3-18.

44. Krieger N. Theories for social epidemiology in the $21^{\text {st }}$ century: an ecosocial perspective. Int J Epidemiol 2001; 30: 668-77.

45. Barata RB. Epidemiologia Social. Rev Bras Epidemiol, 2005; 8(1): 7-17.

46. Pearce N. Traditional epidemiology, modern epidemiology and public health 1996; 86: 678-83.

47. Barreto M. Por uma epidemiologia da saúde coletiva. Rev Bras Epidemiol 1998; 1(2): 104-30.

48. Borrel C. Métodos utilizados no estudo das desigualdades sociais em Saúde. In: Barata R, organizador. Condições de vida e saúde. Rio de Janeiro: ABRASCO; 1997. pp. 16795.

49. Silva JB, Barros MBA. Epidemiologia e desigualdade: notas sobre a teoria e a história. Pan Am J Public Health 2002; 12(6): 375-83.

50. Kunst AE. Cross-national comparisons of socio-economic differences in mortality. [thesis]. Rotterdam: Erasmus University; 1997.

51. Schneider C, Castillo-Salgado C, Bacallao J, Loyola E, Mujica OJ, Vidaurre M, Roca A. Métodos de medición de las desigualdades de salud. Pan Am J Public Health 2002; 12(6): 398-414.

52. Krieger N, Moss N. Accounting for the public's health: an introduction to selected papers from a US conference on "Measuring social inequalities in health. Int J Health Serv 1996; 26(3):383-90.
53. Muntaner C, Borrell C, Benach J, Pasarín MI, Fernandez E. The association of social class and social stratification with patterns of general and mental health in a Spanish population. Int J Epidemiol 2003; 32(6): 950-8.

54. Barbeau EM, Krieger N, Soobader MJ. Working class matters: socioeconomic disadvantage, race/ehinicity, gender, and smoking in NHIS 2000. Am J Public Health 2004; 94(2): 269-78.

55. Jarvis M, Wardle J. Social patterning of individual health behaviours: the case of cigarette smoking. In: Marmot M, Wilkinson RG. Social determinants of health. Oxford: Oxford University Press; 1999. pp. 240-55.

56. Yancey AK, Wold CM, McCarthy WJ, Weber MD, Lee B, Simon PA, Fielding JE. Physical Inactivity and overweight among Los Angeles county adults. Am J Prev Med 2004; 27(2):146-52.

57. Zaitune MAA, Barros MBA, César CLG, Carandina L, Goldbaum M. Fatores associados ao sedentarismo no lazer em idosos, Campinas, SP. Cad Saúde Pública 2007; 23(6): 1329-38.

58. Mendoza-Sassi RA, Beria JU. Prevalence of alcohol use disorders and associated factors: a population-based study using AUDIT in southern Brazil. Addiction 2003; 98(6):799-804.

59. Malyutina S, Bobak M, Kurilovitch D, Nikitin Y, Marmot $M$. Trends in alcohol intake by education and marital status in urban population in Russia between the mid 1980s and the mid 1990s. Alcohol Alcohol 2004; 39(1): 649

60. Caballero B \& Popkin BM (ed) The nutrition transition: Diet and disease in the developing world. California, USA: Academic Press; 2002.

61. Lima-Costa MF. A saúde dos adultos na região metropolitana de Belo Horizonte: um estudo epidemiológico de base populacional. Belo Horizonte: Nespe/Fiocruz/UFMG; 2004.

62. Huisman M, Kunst AE, Mackenbach JP. Inequalities in the prevalence of smoking in the European Union: comparing education and income. Prev Med 2005; 40: $756-64$.

63. Theme-Filha MM, Szwarcwald CL, Souza-Júnior PRB. Socio-demographic characteristics, treatment coverage and self-rated health of individuals who reported six chronic diseases in Brazil, 2003. Cad Saúde Pública 2005; 21 Supl: S43-S53.

64. Barros MBA, César CLG, Carandina L, Torre GD. Desigualdades sociais na prevalência de doenças crônicas no Brasil, PNAD-2003. Ciência e Saúde Coletiva 2006; 11(4): 911-26.

65. Zaitune MAA, Barros MBA, César CLG, Carandina L, Goldbaum M. Hipertensão arterial em idosos: prevalência, fatores associados e práticas de controle no Município de Campinas, Cad Saúde Pública 2006; 22(2): 285-94. 
66. Amorim VMSL, Barros MBA, Cesar CL, Carandina L, Goldbaum M. Fatores associados à não realização do exame de Papanicolaou: um estudo de base populacional no Município de Campinas, São Paulo, Brasil. Cad Saúde Pública 2006: 22(11): 2329-38.

67. Francisco PM, Donalisio MR, Barros MBA, Cesar CL, Carandina L, Goldbaum M. Fatores associados com a vacinação contra influenza em idosos. Rev Panam Salud Publica 2006: 259-64.
68. Najman JM, Toloo G, Siskind V. Socioeconomic disadvantage and changes in health risk behaviours in Australia: 1989-90 to 2001. Bull World Health Organ 2006; 84(12): 976-184

69. Boerma T, Stansfield SK. Health statistics now: are we making the right investments? Lancet 2007; 369: 779-86.

70. Syme L. Historical perspectives: the social determinants of disease - some roots of the movement. Epidemiologic Perspectives \& Innovations 2005; 2: 1-7. 